\title{
$N$-Propargylation of Indolo-Triterpenoids and Their Application in Mannich Reaction
}

\author{
Elmira F. Khusnutdinova $1, * \mathbb{C}$, Anastasiya V. Petrova ${ }^{1,2} \mathbb{D}$, Gulnaz M. Bashirova ${ }^{2}$ \\ and Oxana B. Kazakova ${ }^{1}$ (D) \\ 1 Ufa Institute of Chemistry UFRS RAS, Ufa 450054, 71 pr. Oktyabrya, Russia; pnastya08@mail.ru (A.V.P.); \\ obf@anrb.ru (O.B.K.) \\ 2 Chemical Department, Bashkir State University, Ufa 450076, 32 Validy Str., Russia; bashirova-1998@mail.ru \\ * Correspondence: ElmaH@inbox.ru; Tel.: +7-347-235-6066
}

Received: 16 May 2019; Accepted: 11 June 2019; Published: 13 June 2019

\begin{abstract}
The introduction of the alkynyl moiety to the triterpenic core through a linkage to the indole nitrogen is described. The reaction of $\mathrm{N}$-propargylindoles with $\mathrm{N}$-methylpiperazine using Mannich reaction led to propargylaminoalkynyl-triterpenoids, whose structures were established by NMR spectroscopy.
\end{abstract}

Keywords: triterpenoids; indoles; $N$-propargylation; alkyne; Mannich reaction

\section{Introduction}

During the last few years, the synthetic transformation of natural compounds to prepare biologically active analogues has become a topical course of bioorganic chemistry. Triterpenoids are a large group of natural compounds that possess a broad-spectrum pharmacological activity and represent a biologically active scaffold for chemical transformations because of several key positions available on the molecule [1,2]. One of the trending topics in the chemistry of triterpenoids is the synthesis of various heterocyclic derivatives by the condensation with A-ring, modification of carboxylic group and $\mathrm{C} 3$ position [3].

Among the $N$-heterocycles, the indole ring system is an important structural component in many pharmaceutical agents [4]. 2,3-Indolo-12-oxo-oleanolic acid showed significant inhibition activity towards protein tyrosine phosphatase $1 \mathrm{~B}$ [5]. The $\mathrm{IC}_{50}$ value of ursolic acid modified with 5-methylindole moiety was comparable to doxorubicin [6], and the chloro-derivative of indolo-betulinic acid was found to be very active towards several cancer cell lines [7]. According to previous reports, some indolo-fused lupanes showed $\alpha$-glucosidase inhibitory activity [8-10]. 2,3-Indolouvaol and 2,3-indolo-28-cyanoethoxybetulin showed antiproliferative activity in vitro towards leukemia, lung, and colon cancer cells [11].

The indole cycle can be converted into other ring systems leading to further privileged structures. According to our recent studies, the oxidized 28-oxo-indolo-allobetulone derivatives possess antiviral activity [12]. Oxidation of an aromatic moiety by $\mathrm{H}_{2} \mathrm{O}_{2}$ provides the ursane indoloquinone formation [13].

At the same time, there are no reports about the synthesis of $N$-substituted indole-fused triterpenoids, which in turn opens up the possibility for obtained of new conjugates. For example, in the last decade one of the priority topics in the chemistry of triterpenoids is the synthesis of various alkynyl derivatives with subsequent modification by click-chemistry to produce biologically active compounds bearing a 1,2,3-triazolyl fragment [14,15]. The conjugates obtained by click or Mannich reaction of C-28-propargyl amide or ester derivatives of 2,3-indolo-triterpenic acid possess the anticancer activity [16,17]. 
In this work, the first example of $N$-propargylation of indolo-triterpenoids is described and their application for Mannich reaction is presented.

\section{Results}

At first, the triterpenic indoles were prepared, containing one reactive center for the following propargylation. For this purpose we have modified 2,3-indolo-betulinic acid $\mathbf{1}$ at the E-ring. The reduction of carboxylic group with $\mathrm{LiAlH}_{4}$ gave 2,3-indolo-betulin 2, which was dehydrated using phosphorus $(\mathrm{V})$ oxychloride in pyridine to give abeo-derivative 3. 2,3-Indolo-28-oxo-allobetulone 4 was obtained by Wagner-Meerwein rearrangement of compound $\mathbf{1}$ in the presence of formic acid (Scheme 1).

Then the reaction of E-ring modified indoles 3 and 4 with propargyl bromide and $\mathrm{NaH}$ in DMF provided the $N$-substituted derivatives 5 and $\mathbf{6}$ with 68 and $70 \%$ yields. The structure of compounds $\mathbf{5}$ and $\mathbf{6}$ was ascertained by NMR spectroscopy. Thus, the disappearance of signals of amine group protons of indolo-fragment at $\delta 7.71$ and $7.76 \mathrm{ppm}$ was observed. The signals of the acetylene group at $\delta$ 72.2-72.3 ppm $\left({ }^{13} \mathrm{C}-\mathrm{NMR}\right)$ and $\delta 1.88$ and $2.38 \mathrm{ppm}\left({ }^{1} \mathrm{H}-\mathrm{NMR}\right)$, as well as methylene $(\delta 5.02-5.04 \mathrm{ppm}$ $\left.\left({ }^{1} \mathrm{H}-\mathrm{NMR}\right)\right)$ were characteristic (see Supplementary Materials).

The reaction of $\mathrm{N}$-propargylindoles 5 and $\mathbf{6}$ with $\mathrm{N}$-methylpiperazine using Mannich reaction (secondary amine, paraformaldehyde, $\mathrm{NaOAc}, \mathrm{CuI}$ ) gave indolo- $\mathrm{N}$-methylpiperazine conjugates 7 and 8 with 72 and $77 \%$ yields. The ${ }^{1} \mathrm{H}-\mathrm{NMR}$ spectra of Mannich bases 7 and 8 showed typical signals of the $\mathrm{N}$-methylpiperazine fragment: the methyl group as a singlet at $\delta 2.20-2.40 \mathrm{ppm}$ and the methylene groups as a multiplet at $\delta 2.38-2.78$ and $2.44-2.80 \mathrm{ppm}$. The signals of the acetylene were observed at $\delta$ 70.9-81.0 ppm ( $\left.{ }^{13} \mathrm{C}-\mathrm{NMR}\right)$.

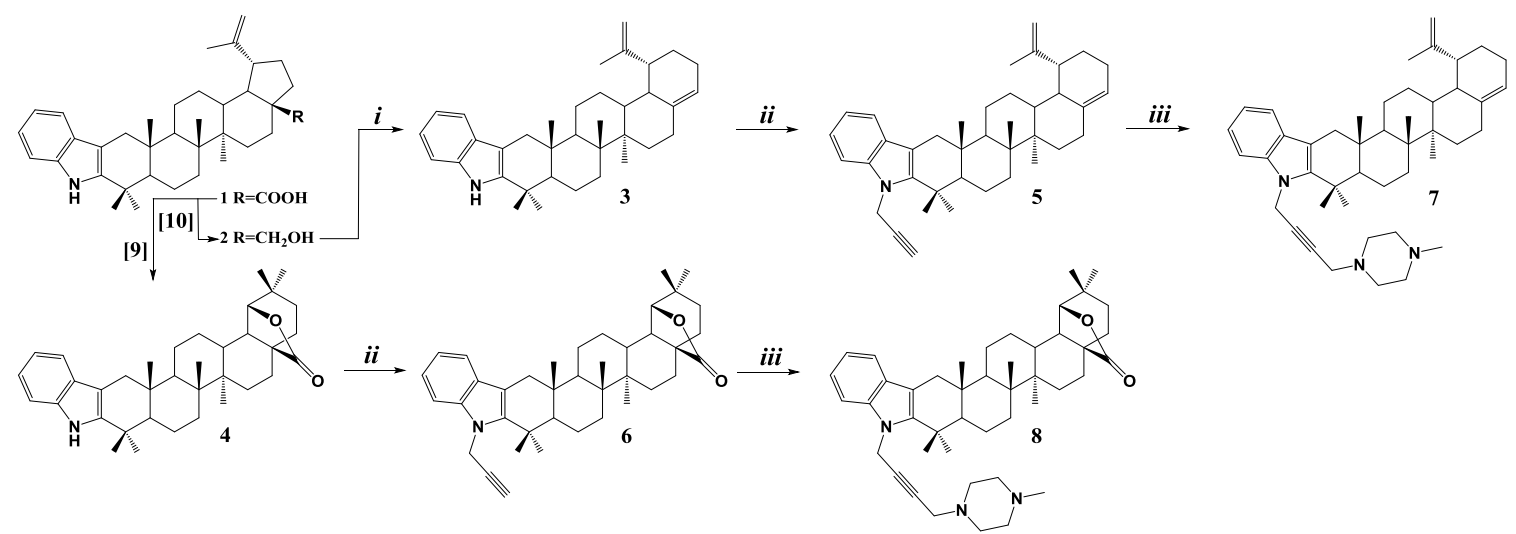

Scheme 1. Synthesis of propargylaminoalkynyl-triterpenoids. Reagent and conditions: i. $\mathrm{POCl}_{3}$, pyridine, reflux, 8 h; ii. Propargyl bromide, NaH, DMF, $0-5{ }^{\circ} \mathrm{C}, 2 \mathrm{~h}$; iii. $\mathrm{N}$-methylpiperazine, paraformaldehyde, $\mathrm{NaOAc}, \mathrm{CuI}, 60^{\circ} \mathrm{C}, 10 \mathrm{~h}$.

\section{Materials and Methods}

The spectra were recorded at the Center for the Collective Use 'Chemistry' of the Ufa Institute of Chemistry, part of the Ufa Federal Research Centre of the Russian Academy of Sciences. ${ }^{1} \mathrm{H}$ and ${ }^{13} \mathrm{C}-\mathrm{NMR}$ spectra were recorded on a "Bruker AM-500" (Bruker, Billerica, MA, USA, 500 and $125.5 \mathrm{MHz}$ respectively, $\delta, \mathrm{ppm}, \mathrm{Hz}$ ) in $\mathrm{CDCl}_{3}$, internal standard tetramethylsilane. Mass spectra were obtained on a liquid chromatograph-mass spectrometer LCMS-2010 EV (Shimadzu, Kyoto, Japan). Melting points were detected on a micro table "Rapido PHMK05" (Nagema, Dresden, Germany). Optical rotations were measured on a polarimeter "Perkin-Elmer 241 MC" (PerkinElmer, Waltham, MA, USA) in a tube length of $1 \mathrm{dm}$. Elemental analysis was performed on a Euro EA-3000 CHNS analyzer (Eurovector, Milan, Italy); the main standard is acetanilide. Thin-layer chromatography analyses were performed on Sorbfil plates (Sorbpolimer, Krasnodar, Russian Federation), using the solvent system chloroform-ethyl acetate, 40:1. Substances were detected by a 10\% solution of a sulfuric acid solution with subsequent 
heating at $100-120^{\circ} \mathrm{C}$ for $2-3 \mathrm{~min}$. Compounds 1 [9] and 4 [9], 2 [11] were obtained according to the methods described previously.

\section{1. [3,2b]Indolo-lup-20(29),17(28)-dien (3)}

A mixture of compound $2(0.51 \mathrm{~g} ; 1 \mathrm{mmol})$ and $\mathrm{POCl}_{3}(0.09 \mathrm{~mL} ; 1 \mathrm{mmol})$ in pyridine $(15 \mathrm{~mL})$ was refluxed for $8 \mathrm{~h}$, then poured into $50 \mathrm{~mL}$ of water and the precipitate was filtered off, and washed with water until neutral $\mathrm{pH}$. The residue was purified by $\mathrm{Al}_{2} \mathrm{O}_{3}$ column chromatography using petroleum ether as eluent to afford compound 3 as a white crystals $(0.42 \mathrm{~g}, 85 \%)$ : $[\alpha]_{D}^{20}+7$ (c $\left.0.75, \mathrm{CH}_{2} \mathrm{Cl}_{2}\right)$, m.p. $207^{\circ} \mathrm{C} .{ }^{1} \mathrm{H}-\mathrm{NMR}\left(\delta, \mathrm{ppm}, \mathrm{CDCl}_{3}, 500 \mathrm{MHz}\right): 7.71(1 \mathrm{H}, \mathrm{br.s}, \mathrm{NH}), 7.51-7.10(4 \mathrm{H}, \mathrm{m}$, arom $), 5.44(1 \mathrm{H}, \mathrm{s}$, $\mathrm{H}-28), 4.72$ and $4.81(2 \mathrm{H}$, both $\mathrm{s}, J=2.0 \mathrm{~Hz}, \mathrm{H}-29), 2.91-2.88\left(2 \mathrm{H}, \mathrm{m}, \mathrm{CH}_{2}\right), 2.31-1.01\left(18 \mathrm{H}, \mathrm{m}, \mathrm{CH}, \mathrm{CH}_{2}\right)$, $1.89(3 \mathrm{H}, \mathrm{s}, \mathrm{H}-30), 1.32,1.24,1.15,1.09,0.92(15 \mathrm{H}$, all s, 5CH 3$) .{ }^{13} \mathrm{C}-\mathrm{NMR}\left(\delta, \mathrm{ppm}, \mathrm{CDCl}_{3}, 125.5 \mathrm{MHz}\right)$ : 151.0 (C-20), 141.7 (C-17), 140.9 (C-arom), 136.2 (C-arom), 128.4 (C-arom), 120.9 (C-28), 118.9 (C-arom), 118.6 (C-arom), 118.0 (C-arom), 110.4 (C-29), 108.9 (C-arom), 107.1 (C-arom), 55.9, 53.4, 49.4, 46.6, 44.5, 42.4, 40.7, 38.3, 37.4, 36.7, 34.2, 33.9, 33.5, 32.8, 30.9, 27.9, 26.9, 23.9, 22.6, 21.9, 21.4, 19.3, 16.6, 15.8. Anal. Calcd for $\mathrm{C}_{36} \mathrm{H}_{49} \mathrm{~N}$ : C, 87.21; H, 9.96. Found: C, 87.22; H, 9.97. MS (APCI): $m / z[\mathrm{M}+\mathrm{H}]^{+}$497.80, calcd for $\mathrm{C}_{36} \mathrm{H}_{49} \mathrm{~N}: 496.79$.

\section{2. [3,2b]Indolo-N-prop-2-yn-1-yl-lup-20(29),17(28)-dien (5)}

$\mathrm{NaH}(0.028 \mathrm{~g} ; 1.1 \mathrm{mmol})$, after washing with dry hexane, was suspended in dry DMF (5 mL) followed by the addition of compound $3(0.50 \mathrm{~g} ; 1 \mathrm{mmol})$. After the color change of the reaction mixture, propargyl bromide $(0.178 \mathrm{~g} ; 1.5 \mathrm{mmol})$ was added drop-wise. Reaction mixture was stirred for $2 \mathrm{~h}$ at $0-5{ }^{\circ} \mathrm{C}$. After the completion of the reaction, ice cooled water $(10 \mathrm{~mL})$ was added to the reaction mixture and extracted with $\mathrm{CH}_{2} \mathrm{Cl}_{2}(3 \times 15 \mathrm{~mL})$. The combined organic layers were then washed with water $(3 \times 50 \mathrm{~mL})$ and dried over $\mathrm{CaCl}_{2}$. Organic phase was concentrated under vacuum and the crude was purified by flesh chromatography (eluent petroleum ether- $\mathrm{Et}_{2} \mathrm{O}(3: 1)$ to procedure compound 5 as a yellow powder $(0.36 \mathrm{~g}, 68 \%)$ : $[\alpha]_{D}^{20}+36\left(\mathrm{c} 0.75, \mathrm{CH}_{2} \mathrm{Cl}_{2}\right), \mathrm{m} . \mathrm{p} .195-197^{\circ} \mathrm{C} .{ }^{1} \mathrm{H}-\mathrm{NMR}(\delta, \mathrm{ppm}$, $\left.\mathrm{CDCl}_{3}, 500 \mathrm{MHz}\right): 7.48-7.05(4 \mathrm{H}, \mathrm{m}, \mathrm{arom}), 5.40(1 \mathrm{H}, \mathrm{s}, \mathrm{H}-28), 5.04\left(2 \mathrm{H}, \mathrm{s}, \mathrm{CH}_{2}\right), 4.78-4.70(2 \mathrm{H}$, both s, $J=2.0 \mathrm{~Hz}, \mathrm{H}-29), 2.90-2.84\left(2 \mathrm{H}, \mathrm{m}, \mathrm{CH}_{2}\right), 2.38(1 \mathrm{H}, \mathrm{s}, \mathrm{C} \equiv \mathrm{C} \underline{\mathrm{H}}), 2.23-0.95\left(21 \mathrm{H}, \mathrm{m}, \mathrm{CH}, \mathrm{CH}_{2}\right), 1.80(3 \mathrm{H}$, s, H-30), 1.40, 1.21, 1.13, 1.05, $0.90\left(15 \mathrm{H}\right.$, all s, $\left.5 \mathrm{CH}_{3}\right) .{ }^{13} \mathrm{C}-\mathrm{NMR}\left(\delta, \mathrm{ppm}, \mathrm{CDCl}_{3}, 125.5 \mathrm{MHz}\right): 151.0$ (C-20), 141.7 (C-17), 140.9 (C-arom), 136.1 (C-arom), 128.3 (C-arom), 120.9 (C-28), 118.9 (C-arom), 118.5 (C-arom), 117.9 (C-arom), 110.4 (C-29), 108.9 (C-arom), 107.1 (C-arom), 80.1 (C-38), 72.2 (C-39), 55.4, 53.3, 49.4, 46.5, 44.4, 42.3, 40.9, 38.3, 37.4, 34.8, 33.9, 32.7, 30.9, 30.4, 29.5, 28.3, 26.8, 23.8, 23.2, 22.5, 21.6, 19.3, 16.6, 15.7, 14.9. Anal. Calcd for $\mathrm{C}_{39} \mathrm{H}_{51} \mathrm{~N}$ : C, 87.75; H, 9.63. Found: C, 87.72; H, 9.65. MS (APCI): $m / z[\mathrm{M}+\mathrm{H}]^{+}$535.82, calcd for $\mathrm{C}_{39} \mathrm{H}_{51} \mathrm{~N}: 534.84$.

\section{3. [3,2b]Indolo-N-prop-2-yn-1-yl-28-oxo-allobetulon (6)}

Compound 6 was obtained from compound $4(0.53 \mathrm{~g} ; 1 \mathrm{mmol})$ by a procedure similar to the synthesis of compound 5 as a yellow powder $(0.40 \mathrm{~g}, 70 \%)$ : $[\alpha]_{D}^{20}+80\left(\mathrm{c} 0.75, \mathrm{CH}_{2} \mathrm{Cl}_{2}\right)$, m.p. $156^{\circ} \mathrm{C}$. ${ }^{1} \mathrm{H}-\mathrm{NMR}\left(\delta, \mathrm{ppm}, \mathrm{CDCl}_{3}, 500 \mathrm{MHz}\right): 7.49-7.02(4 \mathrm{H}, \mathrm{m}, \operatorname{arom}), 5.02\left(2 \mathrm{H}, \mathrm{s}, \mathrm{CH}_{2}\right), 4.02(1 \mathrm{H}, \mathrm{s}, \mathrm{H}-19)$, 2.92-2.82 (2H, m, $\left.\mathrm{CH}_{2}\right), 1.88(1 \mathrm{H}, \mathrm{s}, \mathrm{C} \equiv \mathrm{C} \underline{\mathrm{H}}), 2.23-1.00\left(20 \mathrm{H}, \mathrm{m}, \mathrm{CH}, \mathrm{CH}_{2}\right), 1.42,1.28,1.18,1.00,0.95$, 0.88, $0.85\left(21 \mathrm{H}\right.$, all s, $\left.7 \mathrm{CH}_{3}\right) .{ }^{13} \mathrm{C}-\mathrm{NMR}\left(\delta, \mathrm{ppm}, \mathrm{CDCl}_{3}, 125.5 \mathrm{MHz}\right): 180.0$ (C-28), 140.9 (C-arom), 136.2 (C-arom), 128.3 (C-arom), 121.4 (C-arom), 118.9 (C-arom), 117.9 (C-arom), 110.4 (C-arom), 106.8 (C-arom), 86.1 (C-19), 78.9 (C-38), 72.3 (C-39), 55.6, 53.5, 50.3, 50.1, 46.7, 38.1, 37.5, 36.2, 34.8, 33.1, 32.9, 32.4, 31.9, 30.9, 29.5, 28.8, 28.0, 26.7, 25.6, 24.0, 23.1, 21.6, 21.4, 19.1, 16.8, 15.4, 13.7. Anal. Calcd for $\mathrm{C}_{39} \mathrm{H}_{51} \mathrm{NO}_{2}$ : C, 82.78; H, 9.09; N, 2.48. Found: C, 82.77; H, 9.08; N, 2.48. MS (APCI): $m / z[\mathrm{M}+\mathrm{H}]^{+}$ 567.83, calcd for $\mathrm{C}_{39} \mathrm{H}_{51} \mathrm{NO}_{2}: 566.82$.

\section{4. [3,2b]Indolo-N-(4-(4-methylpiperazin-1-yl)but-2-yn-1-yl)-lup-20(29),17(28)-dien (7)}

$\mathrm{N}$-methylpiperazine $(0.72 \mathrm{mmol} ; 80 \mu \mathrm{L})$, paraformaldehyde $(0.18 \mathrm{~g} ; 6 \mathrm{mmol}), \mathrm{NaOAc}(0.25 \mathrm{~g}$; $3 \mathrm{mmol})$ and $\mathrm{CuI}(6 \mathrm{mg} ; 0.03 \mathrm{mmol})$ were added to a solution of compound $5(0.32 \mathrm{~g} ; 0.6 \mathrm{mmol})$ in dry 
dioxane $(12 \mathrm{~mL})$. The reaction mixture was stirred under argon for $10 \mathrm{~h}$ at $60^{\circ} \mathrm{C}$. After the reaction was complete by thin-layer chromatography the mixture was diluted with water and extracted with $\mathrm{CHCl}_{3}(3 \times 20 \mathrm{~mL})$. The combined organic layer was washed with water $(3 \times 50 \mathrm{~mL})$, dried over $\mathrm{CaCl}_{2}$ and evaporated under reduced pressure. The residue was purified by $\mathrm{SiO}_{2}$ column chromatography (eluent $\mathrm{CHCl}_{3}-\mathrm{MeOH}$ 100:0 $\left.\rightarrow 90: 10\right)$ with obtaining of compound 7 as a yellow solid $(0.28 \mathrm{~g}, 72 \%)$, $[\alpha]_{D}^{20}+97\left(\mathrm{c} 0.75, \mathrm{CH}_{2} \mathrm{Cl}_{2}\right)$, m.p. $188-189^{\circ} \mathrm{C} .{ }^{1} \mathrm{H}-\mathrm{NMR}\left(\delta, \mathrm{ppm}, \mathrm{CDCl}_{3}, 500 \mathrm{MHz}\right): 7.48-7.02(4 \mathrm{H}, \mathrm{m}$, arom), $5.38(1 \mathrm{H}, \mathrm{s}, \mathrm{H}-28), 5.02\left(2 \mathrm{H}, \mathrm{s}, \mathrm{CH}_{2}\right), 4.69-4.78(2 \mathrm{H}$, both s, $J=2.0 \mathrm{~Hz}, \mathrm{H}-29), 3.69(1 \mathrm{H}, \mathrm{s}, \mathrm{H}-18)$, $3.21\left(2 \mathrm{H}, \mathrm{s}, \mathrm{CH}_{2}\right), 2.78-2.38\left(8 \mathrm{H}, \mathrm{m}, 4 \mathrm{CH}_{2}\right), 2.40\left(3 \mathrm{H}, \mathrm{s}, \mathrm{NCH}_{3}\right), 2.28-1.15\left(22 \mathrm{H}, \mathrm{m}, \mathrm{CH}, \mathrm{CH}_{2}\right), 1.78(3 \mathrm{H}$, s, H-30), 1.41, 1.32, 1.18, 1.10, $0.89\left(15 \mathrm{H}\right.$, all s, $\left.5 \mathrm{CH}_{3}\right) .{ }^{13} \mathrm{C}-\mathrm{N} M R\left(\delta, \mathrm{ppm}, \mathrm{CDCl}_{3}, 125.5 \mathrm{MHz}\right): 151.0$ (C-20), 141.6 (C-17), 140.4 (C-arom), 137.3 (C-arom), 127.9 (C-arom), 121.2 (C-28), 119.1 (C-arom), 118.5 (C-arom), 117.9 (C-arom), 109.2 (C-29), 108.9 (C-arom), 108.1 (C-arom), 81.0 (C-38), 78.5 (C-39), 55.5, 55.3, 54.2, 50.6, 49.5, 46.8, 46.0, 45.4, 44.8, 42.3, 40.9, 39.7, 37.9, 37.7, 35.0, 34.9, 33.8, 33.5, 32.7, 29.6, 28.3, 26.9, 24.2, 23.8, 22.4, 21.9, 21.7, 19.3, 16.4, 15.7, 14.9. Anal. Calcd for $\mathrm{C}_{45} \mathrm{H}_{63} \mathrm{~N}_{3}: \mathrm{C}, 83.67 ; \mathrm{H}, 9.83 ; \mathrm{N}, 6.50$. Found: $\mathrm{C}, 83.65 ; \mathrm{H}, 9.84 ; \mathrm{N}, 6.53$. MS (APCI): $m / z[\mathrm{M}+\mathrm{H}]^{+}$648.02, calcd for $\mathrm{C}_{45} \mathrm{H}_{63} \mathrm{~N}_{3}: 647.01$.

\section{5. [3,2b]Indolo-N-(4-(4-methylpiperazin-1-yl)but-2-yn-1-yl)-28-oxo-allobetulon (8)}

Compound 8 was obtained from $6(0.34 \mathrm{~g} ; 0.6 \mathrm{mmol})$ by a procedure similar to the synthesis of compound 7 as a yellow resin $(0.31 \mathrm{~g}, 77 \%),[\alpha]_{D}^{20}+17\left(\mathrm{c} 0.75, \mathrm{CH}_{2} \mathrm{Cl}_{2}\right)$, m.p. $165-167^{\circ} \mathrm{C} .{ }^{1} \mathrm{H}-\mathrm{NMR}(\delta$, ppm, $\left.\mathrm{CDCl}_{3}, 500 \mathrm{MHz}\right): 7.45-7.05\left(4 \mathrm{H}, \mathrm{m}\right.$, arom), $5.05\left(2 \mathrm{H}, \mathrm{s}, \mathrm{CH}_{2}\right), 4.00(1 \mathrm{H}, \mathrm{s}, \mathrm{H}-19), 3.22(2 \mathrm{H}, \mathrm{s}$, $\left.\mathrm{CH}_{2}\right), 2.80-2.44\left(8 \mathrm{H}, \mathrm{m}, 4 \mathrm{CH}_{2}\right), 2.20\left(3 \mathrm{H}, \mathrm{s}, \mathrm{NCH}_{3}\right), 2.30-1.10\left(22 \mathrm{H}, \mathrm{m}, \mathrm{CH}, \mathrm{CH}_{2}\right), 1.45,1.32,1.30,1.09$, 1.00, 0.92, $0.88\left(21 \mathrm{H}\right.$, all s, $\left.7 \mathrm{CH}_{3}\right) .{ }^{13} \mathrm{C}-\mathrm{NMR}\left(\bar{\delta}, \mathrm{ppm}, \mathrm{CDCl}_{3}, 125.5 \mathrm{MHz}\right): 179.9(\mathrm{C}-28), 140.4$ (C-arom), 137.3 (C-arom), 127.9 (C-arom), 121.3 (C-arom), 119.2 (C-arom), 117.9 (C-arom), 109.2 (C-arom), 107.9 (C-arom), 85.9 (C-19), 78.2 (C-38), 70.9 (C-39), 55.6, 53.9, 50.3, 46.7, 46.2, 41.7, 40.6, 39.9, 38.1, 37.8, 36.2, 35.0, 34.6, 33.6, 33.1, 32.4, 31.9, 29.7, 29.6, 28.8, 27.9, 26.7, 25.6, 23.9, 21.7, 21.5, 19.4, 18.7, 19.1, 17.0, 16.6, 15.4, 13.7. Anal. Calcd for $\mathrm{C}_{45} \mathrm{H}_{63} \mathrm{~N}_{3} \mathrm{O}_{2}: \mathrm{C}, 79.72 ; \mathrm{H}, 9.37 ; \mathrm{N}, 6.20$. Found: $\mathrm{C}, 79.73 ; \mathrm{H}, 9.36 ; \mathrm{N}, 6.22$. MS (APCI): $m / z[\mathrm{M}+\mathrm{H}]^{+}$680.01, calcd for $\mathrm{C}_{45} \mathrm{H}_{63} \mathrm{~N}_{3} \mathrm{O}_{2}: 679.02$.

\section{Conclusions}

The first synthesis of triterpenic $\mathrm{N}$-propargylindoles and their modification using a $\mathrm{Cu}(\mathrm{I})$-catalyzed Mannich reaction were achieved.

Supplementary Materials: ${ }^{1} \mathrm{H}$ and ${ }^{13} \mathrm{C}$ spectra for compounds are available online.

Author Contributions: E.K. prepared and corrected the manuscript; A.P. and G.B. conducted the experiment, and did structure elucidation and prepared the manuscript; O.K. brought the idea, and managed the research and prepared the manuscript.

Funding: This work was supported by the Ministry of Education of the Russian Federation, State task project no. AAAA-A17-117011910023-2.

Conflicts of Interest: The authors declare no conflict of interest.

\section{References}

1. Salvador, J.A.R.; Leal, A.S.; Valdeira, A.S.; Gonçalves, B.M.F.; Alho, D.P.S.; Figueiredo, S.A.C.; Silvestre, S.M.; Mendes, V.I.S. Oleanane-, ursane-, and quinone methide friedelane-type triterpenoid derivatives: Recent advances in cancer treatment. Eur. J. Med. Chem. 2017, 142, 95-130. [CrossRef] [PubMed]

2. Sousa, J.L.C.; Freire, C.S.R.; Silvestre, A.J.D.; Silva, A.M.S. Recent developments in the functionalization of betulinic acid and its natural analogues: a route to new bioactive compounds. Molecules 2019, 24, 355. [CrossRef] [PubMed]

3. Kvasnica, M.; Urban, M.; Dickinson, N.J.; Sarek, J. Pentacyclic triterpenoids with nitrogen- and sulfur-containing heterocycles: synthesis and medicinal significance. Nat. Prod. Rep. 2015, 32, 1303-1330. [CrossRef] [PubMed] 
4. Kaushik, N.K.; Kaushik, N.; Attri, P.; Kumar, N.; Kim, H.C.; Verma, A.K.; Choi, E.H. Biomedical importance of indoles. Molecules 2013, 18, 6620-6662. [CrossRef]

5. Qiu, W.W.; Shen, Q.; Yang, F.; Wang, B.; Zou, H.; Li, J.Y.; Li, J.; Tang, J. Synthesis and biological evaluation of heterocyclic ring-substituted maslinic acid derivatives as novel inhibitors of protein tyrosine phosphatase $1 \mathrm{~B}$. Bioorg. Med. Chem. Lett. 2009, 19, 6618-6622. [CrossRef]

6. Gu, W.; Hao, Y.; Zhang, G.; Wang, S.F.; Miao, T.T.; Zhang, K.P. Synthesis, in vitro antimicrobial and cytotoxic activities of new carbazole derivatives of ursolic acid. Bioorg. Med. Chem. Lett. 2015, 25, 554-557. [CrossRef] [PubMed]

7. Kumar, V.; Rani, N.; Aggarwal, P.; Sanna, V.K.; Singh, A.T.; Jaggi, M.; Joshi, N.; Sharma, P.K.; Irchhaiya, R.; Burman, A.C. Synthesis and cytotoxic activity of heterocyclic ring-substituted betulinic acid derivatives. Bioorg. Med. Chem. Lett. 2008, 18, 5058-5062. [CrossRef] [PubMed]

8. Khusnutdinova, E.F.; Smirnova, I.E.; Giniyatullina, G.V.; Medvedeva, N.I.; Yamansarov, E.Y.; Kazakov, D.V.; Kazakova, O.B.; Linh, P.T.; Viet, do Q.; Huong, D.T. Inhibition of alpha-glucosidase by synthetic derivatives of lupane, oleanane, ursane and dammarane triterpenoids. Nat. Prod. Commun. 2016, 11, 33-35. [PubMed]

9. Khusnutdinova, E.F.; Smirnova, I.E.; Kazakova, O.B.; Petrova, A.V.; Nguyen, T.T.H. Synthesis and evaluation of 2,3-indolotriterpenoids as new $\alpha$-glucosidase inhibitors. Med. Chem. Res. 2017, 26, 2737-2742. [CrossRef]

10. Khusnutdinova, E.F.; Petrova, A.V.; Nguyen, T.T.H.; Le, T.T.A.; Nguyen, T.T.; Ba, T.C.; Babkov, D.A.; Kazakova, O.B. Structural modifications of 2,3-indolobetulinic acid: Design and synthesis of highly potent $\alpha$-glucosidase inhibitors. Bioorg. Chem. 2019, 88. in press. [CrossRef]

11. Khusnutdinova, E.F.; Petrova, A.V.; Apryshko, G.N.; Kukovinets, O.S.; Kazakova, O.B. Synthesis and cytotoxicity of indole derivatives of betulin, erythrodiol, and uvaol. Russ. J. Bioorg. Chem. 2018, 44, 322-329. [CrossRef]

12. Khusnutdinova, E.F.; Kazakova, O.B.; Lobov, A.N.; Kukovinets, O.S.; Suponitsky, K.Y.; Meyers, C.B.; Prichard, M.N. Synthesis of A-ring quinolones, nine-membered oxolactams and spiroindoles by oxidative transformations of 2,3-indolotriterpenoids. Org. Biomol. Chem. 2019, 17, 585-597. [CrossRef] [PubMed]

13. Hao, Y.; Hua, D.W.; Miao, T.T.; Wang, S.F.; Jin, X.Y.; Gu, W. Synthesis, crystal structure and antitumor activity of a new indolequinone derivative of ursolic acid. Chin. J. Struct. Chem. 2016, 35, 1167-1173.

14. Pokorny, J.; Borkova, L.; Urban, M. Click reactions in chemistry of triterpenes-advance towards development of potential therapeutics. Curr. Med. Chem. 2018, 25, 636-658. [CrossRef] [PubMed]

15. Csuk, R.; Deigner, H.P. The potential of click reactions for the synthesis of bioactive triterpenes. Bioorg. Med. Chem. Lett. 2019, 29, 949-958. [CrossRef] [PubMed]

16. Khusnutdinova, E.F.; Petrova, A.V.; Kukovinets, O.S.; Kazakova, O.B. Synthesis and cytotoxicity of 28-N-propargylaminoalkylated 2,3-indolotriterpenic acids. Nat. Prod. Commun. 2018, 13, 665-668. [CrossRef]

17. Suman, P.; Patel, A.; Solano, L.; Jampan, G.; Gardner, Z.S.; Holt, G.M.; Jonnalagadda, S.C. Synthesis and cytotoxicity of baylis-hillman template derived betulinic acid-triazole conjugates. Tetrahedron 2017, 73, 4214-4226. [CrossRef]

(C) 2019 by the authors. Licensee MDPI, Basel, Switzerland. This article is an open access article distributed under the terms and conditions of the Creative Commons Attribution (CC BY) license (http://creativecommons.org/licenses/by/4.0/). 